\title{
Resolution and systematic limitations in beam-based alignment
}

\author{
P. Tenenbaum and T. O. Raubenheimer \\ Stanford Linear Accelerator Center, Stanford University, Stanford, California 94309
}

(Received 23 March 2000; published 25 May 2000)

\begin{abstract}
Beam-based alignment of quadrupoles by variation of quadrupole strength is a widely used technique in accelerators today. We describe the dominant systematic limitation of such algorithms, which arises from the change in the center position of the quadrupole as the strength is varied, and derive expressions for the resulting error. In addition, we derive an expression for the statistical resolution of such techniques in a periodic transport line, given knowledge of the line's transport matrices, the resolution of the beam position monitor system, and the details of the strength variation procedure. These results are applied to the Next Linear Collider main linear accelerator, an $11 \mathrm{~km}$ accelerator containing 750 quadrupoles and 5000 accelerator structures. We find that, in principle, a statistical resolution of $1 \mu \mathrm{m}$ is easily achievable, but the systematic error due to variation of the magnetic centers could be several times larger.
\end{abstract}

PACS numbers: 29.27.Eg

\section{INTRODUCTION}

Modern high-energy particle accelerators use quadrupole magnets to focus the charged particle beams. In most accelerators, the alignment of these quadrupole magnets is a prerequisite to achieving the performance goals; most measures of beam quality (transmission, emittance, polarization) are degraded by poor alignment. The best conventional mechanical alignment techniques typically have errors larger than $\sim 100 \mu \mathrm{m}$ due to the limited line of sight and errors fiducializing the components. This level of alignment is insufficient for many operating accelerators, and future accelerators will require even better alignment as requirements on beam quality become more stringent. Two examples of future accelerators are the Linac Coherent Light Source [1], a free-electron laser, which will generate coherent $x$ rays using a $15 \mathrm{GeV}$ electron beam and will require beam-to-quad alignment at the level of $5 \mathrm{~m}$, and the Next Linear Collider (NLC) [2], a future linear collider, which will require quadrupole alignment at the level of $1-2 \mu \mathrm{m}$.

To attain micron-level alignment, it is necessary to use the beam as the alignment tool. In recent years, several accelerators have developed various beam-based alignment techniques. All these techniques are variations on a common approach in which the strength of a quadrupole is varied, causing a dipole deflection of an offset beam, and then the downstream deflection is measured as a function of the quadrupole strength to determine the beam-to-quad offset.

The statistical error of these beam-based approaches arises from the finite resolution of the beam position measurement devices (BPMs). With the development of BPMs having submicron resolution, the statistical errors can be very small and, furthermore, the errors can be reduced with additional data. In contrast, systematic errors will cause the algorithm to converge to an incorrect so- lution and may provide a more fundamental limitation. Fortunately, the quadrupole variation beam-based alignment algorithms are nulling techniques; if the beam passes through the center of the quadrupole, no deflection is produced. Therefore, any systematic errors which distort the fitted magnitude of the deflection will be decreased by iterating the procedure, provided the errors are sufficiently small that the algorithm converges. Examples of these modeling errors are errors in the strength of the varied quadrupole, errors in the transport matrix from the quad to the BPMs (including errors in the energy or energy gain of the beam line), and errors in the BPM scale factors.

One important class of systematic error which is not addressed by iteration of the algorithms is a shift in the center position of the quadrupole when its strength is varied. These shifts could be caused by thermal effects, mechanical distortion of the magnet, nonuniform coil placement, leakage currents in the coils, unequal permeabilities, or remnant fields in the four pole pieces which comprise the magnet. This variation of the magnetic center may be the fundamental limitation to the resolution of beam-based alignment via quadrupole variation, except in certain extreme cases (for example, ballistic alignment, described below).

In this paper, we discuss the effect of these systematic errors as well as the statistical errors. We will concentrate on the effects in a long transport line or accelerator with roughly periodic beta functions and uniformly spaced quadrupoles; the results can be easily generalized to other lattices. We will start by briefly reviewing some of the different quadrupole alignment techniques. Next, we discuss the statistical and systematic errors and then apply these results to the alignment of the NLC main linac where we estimate the magnitude of some of these sources of magnetic center variation. Finally, we used the measured variation in the quadrupole magnets of the 
final focus test beam (FFTB) [3] to estimate the shifts in the magnetic center and the effect on the NLC alignment procedure.

Last, it should be noted that an alternate approach to the quadrupole variation technique is to vary the beam energy. However, in most systems, it is much easier to vary the quadrupole strength in a well-controlled manner than to vary the beam energy. In addition, the analysis of data is simpler because variation of the quad strength allows a subset of quads to be varied (ideally, one quad at a time) while variation of the energy affects the focusing strength of all downstream quads. We will not address this technique further in this paper except to note that the beam energy variation methods also have a systematic limitation which is similar to the variation of the magnetic centers, namely, rf deflections which are transverse deflections that depend on the accelerating fields in the accelerator structures.

\section{BEAM-BASED ALIGNMENT TECHNIQUES}

As discussed, all of the quadrupole beam-based alignment techniques are variations on a common approach: change the quadrupole strength (or the beam energy) and measure the resulting deflection. However, over the past decade, a number of different implementations have been developed. In this section we will briefly introduce some of these different solutions, concentrating on those applicable to transport lines or linear accelerators.

\section{A. Stanford Linear Collider (SLC) injector linac}

The Stanford Linear Collider (SLC) injector linac accelerates bunches of electrons and positrons from 0.2 to $1.2 \mathrm{GeV}$ for injection into the damping rings. At this low energy, these large emittance beams occupy a significant fraction of the available aperture and, to constrain the beam sizes, the linac uses a dense array of 62 quadrupoles to transport the beams. Most of these quadrupoles are very large aperture magnets that are mounted around the accelerator structures. This mounting system tends to lead to relatively large alignment errors. To make the situation worse, the BPMs are located only between the accelerator structures, and thus there are many more magnets than BPMs and dipole correctors, which makes the trajectory correction difficult. In order to measure misalignments of the quadrupoles, a pair of consecutive quadrupoles was reduced from nominal to zero strength, and the resulting deflection was measured on downstream BPMs [4]; pairs of magnets were used to limit the beta beats which would increase the beam size and cause beam loss. By advancing from upstream to downstream (i.e., first quads 1 and 2 are off, then quads 2 and 3, etc.), it was possible to measure the misalignment of each quad in the horizontal and vertical planes. This technique is similar to ballistic alignment (see below) in that it avoids the systematic limitation of changes in the quadrupole center as a function of magnet strength by completely deactivating the magnets that are to be aligned.

\section{B. SLC main linac: two-beam alignment}

The SLC main linac accelerates damped electron and positron beams from 1.2 to $46.6 \mathrm{GeV}$, and contains 275 quadrupoles. Here, many quadrupoles are powered together in strings, and thus individual variation of the magnets was not possible. In one technique, misalignments were measured by observing the difference between the positron and electron trajectories [5]. In this fashion, the linac quads were "varied" by $200 \%$ without any actual change in magnet strength. This technique was used to identify magnets with the largest errors to which the alignment crews could be directed. A similar technique that was developed is the two-beam dispersion free steering (DFS) which simply steered the beam to minimize the effect of the magnet misalignments; this is discussed below.

\section{Dispersion free steering}

In a transport line, misaligned quadrupoles dilute the emittance primarily by introducing dispersion. In the DFS technique, the dispersion is measured directly by simultaneously varying all of the quadrupoles by a fixed fraction of their design strength and measuring the change in the beam trajectory. A steering solution is then generated which simultaneously minimizes the absolute BPM readings and the change in BPM readings with quad strength [6]. The DFS algorithm was tested at the SLC although performance limitations were not fully understood [7]. A more successful recent experiment utilized a combination of the two-beam and DFS techniques, in which the DFS formalism was used to minimize the difference between the electron and positron trajectories in the SLC main linac [8]. This eliminated the dispersive contribution from the misalignments without requiring an access to actually realign the components and appeared to improve the SLC performance significantly. Neither the original two-beam steering algorithm nor two-beam DFS is sensitive to the change in quadrupole center position as a function of quad strength, since neither technique varies the quadrupole strengths. Since both of these techniques require beams of opposite sign traveling in the same direction through the accelerator, the applicability of these techniques is limited.

\section{Final focus test beam}

The alignment algorithm of the FFTB is close to the "classical" beam-based alignment technique: a set of 3 to 6 consecutive quads would be varied in strength, one at a time, and their misalignments were fitted by measuring the trajectory at each strength. The quads would then 
be physically moved onto the electron beam line by a set of precision translation stages. The FFTB alignment achieved a resolution of the beam-to-quad offset which was typically under $10 \mu \mathrm{m}$ in the vertical plane, and under $30 \mu \mathrm{m}$ for all quadrupoles [9].

\section{E. Next Linear Collider main linac}

The NLC main linac design contains 750 quadrupoles in a FODO lattice; each quadrupole contains a high precision BPM and is supported on a precision translation stage similar to those used in the FFTB. The NLC alignment technique envisions using quadrupole variation to measure the beam-to-quad offset of each quad; the offset is then added to the BPM reading to determine the offset between the BPM electrical center and the quadrupole magnetic center. Once the full set of BPM offsets is determined, the movers are employed to steer the linac so as to minimize emittance dilution [10]. Because of the high beam power and the potential damage the beams could cause, a goal of the NLC algorithm is to be a relatively small perturbation to the collider operation; this would allow the alignment to be completed without interference with the machine protection system and, in the most ideal scenario, the realignment might be performed during luminosity production.

\section{F. Ballistic alignment}

The proposed alignment technique of the Compact Linear Collider main linac is to deactivate 10 to 20 consecutive quadrupoles and use a single corrector coil to steer through the resulting field-free region. The quadrupoles are then restored to their design values and the beam is steered to recover the BPM readings obtained with the magnets off [11]. This technique was proposed to address the primary systematic limitation discussed in this paper, namely, the shift of the quadrupole magnetic centers with excitation; however, there are concerns regarding the difficulties associated with controlling the beam with the quadrupoles turned off and limitations that arise from the remnant fields in the quads.

\section{G. $K$ modulation}

Finally, another approach that is worth mentioning is the strength modulation technique. In $K$ modulation, the strength of a quadrupole is varied harmonically at a frequency that is low compared to the betatron and synchrotron frequencies of a storage ring; this generates oscillations in the closed orbit at the same frequency. Because $K$ modulation causes narrow band orbit oscillations, it is possible to use lock-in amplifiers and other techniques to vastly improve the signal-to-noise performance of the measurement [12]. This technique has been used in a number of storage rings where it is not possible to make large changes to the quadrupole strengths because of the effect on the ring tunes; however, the technique is not as applicable to transport lines which have beam pulses at relatively low rates.

\section{STATISTICAL RESOLUTION LIMIT}

Before discussing the systematic limitations, it is worth calculating the statistical resolution of the algorithms. The statistical resolution is usually limited by the BPM resolution, the number of BPMs available for data acquisition, the degree of quadrupole variation the accelerator will tolerate, the amount of data which can be managed by the processing algorithm, and the amount of time available for the measurement. In this section, we will calculate the resolution of the NLC alignment algorithm; a similar analysis was performed for the DFS and the twobeam alignment techniques in Ref. [13].

Consider a system in which the strength of a single quadrupole is varied and a number of downstream BPMs are read for several beam pulses. If a quadrupole with a nominal integrated strength of $K_{q}$ (dimensions $\mathrm{m}^{-1}$ ) is changed in steps of $d K_{q}$ over a series of steps indexed by $j$, such that the integrated strength on any given step is $K_{q}+j d K_{q}$, then the change in the reading of the $i$ th downstream BPM on step $j$ is given by

$$
d x(i, j)=x_{0} j d K_{q} R_{12}(i),
$$

where $R(i)$ is the linear transport matrix from the quad to the $i$ th BPM and $x_{0}$ is the offset between the beam centroid and the magnetic center of the quad. If the resolution of the beam position monitors is given by $\sigma_{\mathrm{BPM}}$, and $m$ beam pulses are read out at each setting of the quadrupole, the statistical resolution with which $x_{0}$ may be fitted is given by

$$
\sigma_{x}^{2}=\frac{\sigma_{\mathrm{BPM}}^{2}}{m \sum_{i} \sum_{j} j^{2}\left(d K_{q}\right)^{2} R_{12}^{2}(i)} .
$$

In a periodic transport line, we can simplify Eq. (2) by replacing $\sum_{i} R_{12}^{2}(i)$ with $n \mathcal{R}_{2}$, where $\mathcal{R}_{2}$ is the sum of $R_{12}^{2}(i)$ over one-half of a betatron wavelength, and $n$ is the number of betatron half-wavelengths which are used. In addition, let us define $j_{m}$ as the value of $j$ with the maximum absolute value, and the maximum change in quadrupole strength $\Delta K_{q} \equiv j_{m} d K_{q}$. In this case,

$$
\sigma_{x}^{2}=\frac{\sigma_{\mathrm{BPM}}^{2}}{n m\left(\Delta K_{q}\right)^{2} \mathcal{R}_{2} \sum_{j}\left(\frac{j}{j_{m}}\right)^{2}} .
$$

In practice, the value of $\mathcal{R}_{2}$ is determined by the requirements of the nominal beam line optics and is not generally a parameter which may be tuned to optimize the beambased alignment procedure; the maximum quadrupole strength excursions $\Delta K_{q}$ are set by the maximum distortion of the beam size tolerable by the downstream optics; $n$ is determined by the length of the beam line or the length over which the model is well known, since errors in the model tend to accumulate over the length of the 
beam line; $m$ is determined by the data acquisition and analysis system and the maximum time allowed for alignment; and $\sigma_{\mathrm{BPM}}$ is a function of the BPM electronics and pickup design.

Note that Eq. (3) does not take into account jitter in the position or angle of the incoming beam at the upstream face of the quadrupole which is being varied; if this jitter is large relative to the BPM resolution, it must be fitted using the upstream BPMs and projected onto the downstream BPMs through the varied quadrupole, which causes a reduction in the precision of the fit of $x_{0}$. If we assume that the beam line is symmetric about the varied quadrupole and that the number of upstream BPMs used to fit the incoming trajectory is equal to the number of downstream BPMs used to fit the kick at the varied quadrupole, the fit error becomes

$$
\sigma_{x}^{2}=\frac{\sigma_{\mathrm{BPM}}^{2}}{n m}\left[\frac{1}{n_{j} \mathcal{R}_{1}}+\frac{2}{\Delta K_{q}^{2} \mathcal{R}_{2} \sum_{j}\left(\frac{j}{j_{m}}\right)^{2}}\right],
$$

where $\mathcal{R}_{1}$ is the sum over one-half a betatron wavelength of $R_{11}^{2}$ from the varied quadrupole to the upstream BPMs and $n_{j}$ is the number of distinct $j$ values used (i.e., $m n_{j}$ is the total number of beam pulses used in the fit). The first term arises from the error in beam position at the quadrupole, while the second term is a combination of the fit error from Eq. (3) and the effect of angle jitter at the quadrupole magnet. For a periodic transport line, $\mathcal{R}_{1}$ is of order unity, while $1 /\left(d K_{q}^{2} j^{2} \mathcal{R}_{2}\right)$ is generally much larger, and thus the second term dominates.

Note that, whatever the source, the statistical error is simply proportional to the BPM resolution and it decreases with additional data. Assuming that the second term in Eq. (4) dominates, we can examine the combination of quadrupole steps and averaging that will minimize the error. Figure 1 shows the value of $\left[\sum_{j}\left(j / j_{m}\right)^{2}\right]^{-0.5}$

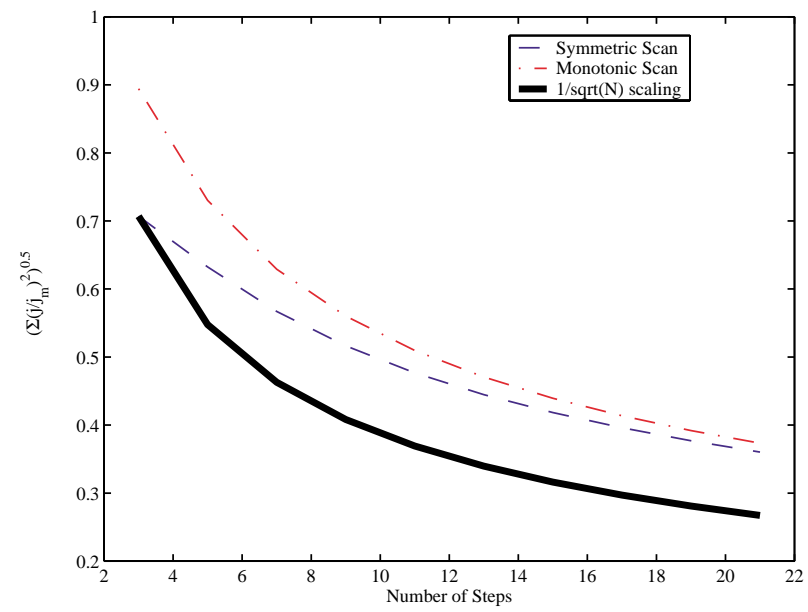

FIG. 1. (Color) $\left[\sum\left(j / j_{m}\right)^{2}\right]^{-0.5}$ as a function of the number of steps. Both symmetric scans (blue dashed line) and monotonic scans (red dot-dashed line) are considered. Also shown is the expected behavior of symmetric scans for $1 /(\text { number steps })^{0.5}$ scaling (black heavy solid line). as a function of the total number of strength steps used in the fit. Note that, for a given value of $\Delta K_{q}$, monotonic scans will have a larger statistical error than scans which are symmetric about the operating point of the magnet, and that for both monotonic and symmetric scans the statistical error falls more slowly than the square root of the reciprocal of the number of steps. Since the data acquisition time and the fitting time are both proportional to the product of the number of steps and the number of pulses per step, the most efficient data acquisition model is to use the largest value of $\Delta K_{q}$ that the beam line will tolerate, the smallest number of strength steps, and the largest number of pulses per step which can be accommodated.

Finally, the analysis described here assumes a fairly accurate model of the beam line optics. This is usually a good assumption over a few betatron wavelengths and, as discussed below, the effect of these modeling errors will decrease with iteration of the alignment algorithm. However, it would also be possible to use the "model independent analysis" techniques discussed in Ref. [14] to attain the desired resolution without any dependence on the optics model if so desired.

\section{SYSTEMATIC RESOLUTION LIMIT}

Now let us consider the systematic limitations. As mentioned previously, the quadrupole variation beambased alignment algorithms are nulling techniques and thus systematic errors which distort the fitted magnitude of the deflection will be reduced by iterating the procedure; this includes all of the optics modeling errors. One systematic error which is not addressed by iteration of the algorithms is the shift in the magnetic center of the quad while its strength is varied. Consider a quadrupole at which the offset between the beam and the quad center at the nominal strength is $x_{D}$, and for which the magnetic center moves a distance $x_{j}$ at step $j$ of the quad varying operation; $x_{j} \equiv 0$ for $j=0$. The absolute deflection at a downstream BPM on step $j$ is given by

$$
x(i, j)=\left(x_{D}+x_{j}\right)\left(K_{q}+j d K_{q}\right) R_{12}(i),
$$

while the deflection at step $j$ relative to the deflection at nominal strength, $x(i, j)-x(i, 0)$, is given by

$$
d x(i, j)=R_{12}(i)\left[\left(x_{D}+x_{j}\right) j d K_{q}+x_{j} K_{q}\right] .
$$

If we fit the measured deflections in Eq. (6) to a model which ignores motion of the magnetic center, i.e., $x_{j}$ is assumed to be zero for all values of $j$, the resulting measured center position will differ from the true position by

$$
x_{M}-x_{D}=\frac{\sum_{j} j^{2} x_{j}}{\sum_{j} j^{2}}+\frac{K_{q}}{d K_{q}} \frac{\sum_{j} j x_{j}}{\sum_{j} j^{2}} .
$$

Equation (7) indicates that the sensitivity to the motion of the quadrupole center is amplified by the fractional quadrupole variation, $K_{q} / d K_{q}$. In general, the error in 
the fit is minimized by scanning the quadrupole strength as close to zero as possible. As an extreme example, consider the special case in which a quadrupole is reduced in a single step from its design strength to zero strength. In this case, $d K_{q}=K_{q}, j=\{0,-1\}$, and Eq. (7) indicates that $x_{M}-x_{D}=0$, regardless of the value or evolution of $x_{j}$. This is similar to the scheme proposed in the ballistic alignment method, although there are other problems, such as controlling the beam size, when the quadrupole strengths become very small.

\section{A. Fitting the center motion}

At this point, we consider the possibility of fitting the motion of the magnetic center as well as the misalignment. For small variations in the magnetic strength, the motion of the magnetic center is likely to be linear in the quadrupole strength. In this case, we can replace $x_{j}$ with $j \dot{x}$ and rewrite Eq. (6),

$$
d x(i, j)=R_{12}(i)\left[j\left(x_{D} d K_{q}+\dot{x} K_{q}\right)+j^{2} \dot{x} d K_{q}\right] .
$$

Equation (8) shows that $d x$ varies linearly during the scan due to a nonzero $x_{D}$, but varies both linearly and quadratically during the scan due to a nonzero $\dot{x}$. It is this quadratic behavior which breaks the degeneracy between $x_{D}$ and $\dot{x}$ and permits fitting to a model in which both $x_{D}$ and $\dot{x}$ are parameters to be determined.

A two parameter least-squares fit such as the one described above results in a fit resolution for the beamto-quad offset at the design magnet strength given by

$$
\sigma_{x}^{2}=\frac{\sigma_{\mathrm{BPM}}^{2}}{n m \mathcal{R}_{2} K_{q}^{2}} \frac{\mathcal{K}^{2} \sum_{j} j^{4}+2 \mathcal{K} \sum_{j} j^{3}+\sum_{j} j^{2}}{\mathcal{K}^{4}\left[\sum_{j} j^{4} \sum_{j} j^{2}-\left(\sum_{j} j^{3}\right)^{2}\right]},
$$

where parameters $m, n$, etc., are defined as in Eq. (3) and $\mathcal{K} \equiv d K_{q} / K_{q}$.

Unfortunately, this tends to be a very difficult fit because we are looking to separate the quadratic dependence from the larger linear variation. This makes the fit ill conditioned and error prone. For example, assuming three steps and $\mathcal{K}=5 \%$, this resolution is over an order of magnitude poorer than the statistical error predicted in Eq. (3). Furthermore, if we consider a system with both linear and quadratic motion of the center with quad strength, we can replace $x_{j}$ with $j \dot{x}+j^{2} \ddot{x}$. This yields

$$
\begin{aligned}
d x(i, j)=R_{12}(i)[ & j\left(x_{D} d K_{q}+\dot{x} K_{q}\right) \\
& \left.+j^{2}\left(\dot{x} d K_{q}+\ddot{x} K_{q}\right)+j^{3} \ddot{x} d K_{q}\right] .
\end{aligned}
$$

In this case, $d x$ varies quadratically during the scan due to both $\dot{x}$ and $\ddot{x}$. Equation (10) implies that if $\ddot{x} / \dot{x}>\mathcal{K}$ the quadratic motion will be dominated by $\ddot{x}$. In this case, the two-parameter fit for $x_{D}$ and $\dot{x}$ will converge upon an incorrect value of $\dot{x}$, and hence an incorrect value of $x_{D}$. Adding additional parameters to the fit will not tend to help because the fit will remain ill conditioned and the resulting errors will be large.

\section{B. Examples of center motion}

At this point, we will discuss a few of the possible causes of the motion of the magnetic center. In particular, we will consider errors that change the relative excitation of a pole or the position of a pole; either of these will shift the magnetic center. To calculate the shift of the center, we assume that the quadrupole is constructed from mechanically perfect pole pieces and each pole piece can be considered an equipotential surface for the magnetic scalar potential. In addition, we assume that the magnet length is much greater than the aperture and the field changes are perturbations to the nominal field. In this case, we may use approximations developed by Halbach [15].

In general, the distance between the mechanical center of the quadrupole magnet and the neutral axis (the longitudinal line along which $|\mathbf{B}| \equiv 0$, which is what is meant by "magnetic center" of a quadrupole magnet) is a function of all the excited nonquadrupole multipole terms. If we assume that the neutral axis is close to the mechanical center, we may make the approximation that the shift in the magnetic center is given by the dipole excitation divided by the quadrupole gradient. Defining $\Phi_{q} \equiv\left(\Phi_{1}-\Phi_{2}+\Phi_{3}-\Phi_{4}\right) / 4$, where $\Phi_{n}$ is the scalar potential of each of the four poles, we can calculate the distance between mechanical and magnetic centers due to unequal excitation of the poles,

$$
\Delta y \approx\left(\Phi_{1}+\Phi_{2}-\Phi_{3}-\Phi_{4}\right) \frac{0.199 \sqrt{2}}{2 \Phi_{q}} a_{q},
$$

where $a_{q}$ is the aperture radius of the quadrupole and the factor 0.199 comes from the tables in Ref. [15].

In a similar manner, we can calculate the shift in the magnetic center due to changes in the radial position of the poles,

$$
\Delta y \approx\left(a_{1}+a_{2}-a_{3}-a_{4}\right) \frac{0.425 \sqrt{2}}{2},
$$

where $a_{i}$ is the radial position of the $i$ th pole.

There are a number of different effects that could cause the pole excitation to vary as a function of excitation: two such examples are leakage currents that depend on the coil temperature and thereby excitation or different permeabilities of the poles that change the excitation as the poles approach saturation. The first case is trivial to estimate:

$$
\Delta y \approx \frac{\sqrt{6}}{10}\left(\frac{\Delta I}{I}\right)_{\mathrm{rms}} a_{q},
$$

where $(\Delta I / I)$ is the rms leakage current and we have assumed that the leakage currents are random, with the current loss arising at the midpoint of each coil, and that the poles are powered in series. This effect does not 
cause the center to shift with excitation if the currents are simply proportional to the excitation; however, these small leakage currents are likely to arise from shorts whose resistance will likely vary as the temperature of the coil changes with excitation of the magnet.

The second case is also straightforward to estimate: the change in excitation depends on the degree to which the pole is saturated which is quantified by the core efficiency; this is the deviation of the quadrupole gradient from that with infinite permeability. Again, assuming random variation in the pole permeability, the shift of the magnetic center would be roughly

$$
\Delta y \approx \frac{\sqrt{2}}{5}(1-\eta) a_{q}\left(\frac{\Delta \mu}{\mu}\right)_{\mathrm{rms}},
$$

where $\eta$ is the core efficiency and $(\Delta \mu / \mu)$ is the rms relative variation of the permeability from pole to pole.

Next, situations that cause the radial position of the poles to move are also easy to envision: the most obvious is mechanical deformation of the magnet which arises as the magnetic forces increase with excitation. Two other cases are related to the magnet temperature: if the conductor resistance or cooling differs from pole to pole, the poles will expand different amounts as the excitation, and thereby the coil temperatures, change; alternately, if the magnet is supported on its base, the vertical position of the magnetic center will change as the temperature changes with excitation.

The first case depends upon the detailed mechanical design of the magnet and thus is difficult to estimate; the other two cases are simpler. If the coils have different conductivity or different cooling, the differential expansion will be given by

$$
\Delta y \approx C_{T} \frac{L_{p}}{2} 0.425\left(\Delta T_{p}\right)_{\mathrm{rms}}\left(\frac{\Delta I^{2}}{I^{2}}+\frac{2 \Delta I}{I}\right),
$$

where $C_{T}$ is the coefficient of thermal expansion for iron, $L_{p}$ is the transverse length of the pole piece from tip to flux-return yoke, $\Delta T_{p}$ is the rms difference in the coil temperature rise at nominal operating current, and $\Delta I / I$ is the fractional change in current required for the quadrupole-variation procedure.

Next, assuming that the magnets are in thermal equilibrium and the magnets are supported on their base, the vertical position of the magnetic center would change by roughly

$$
\Delta y \approx C_{T} \frac{L_{m}}{2} \Delta T_{f}\left(\frac{\Delta I^{2}}{I^{2}}+\frac{2 \Delta I}{I}\right),
$$

where $L_{m}$ is the overall transverse size of the magnet, $\Delta T_{f}$ is the average temperature rise at nominal operating current, and other parameters are as above. This could be a large effect; however, the magnet will not usually reach thermal equilibrium in the time required for the alignment and thus the full effect will not be realized.
In all of the previous cases, the motion of the magnetic center will be a nonlinear function of the excitation, which makes the fitting procedures described earlier quite difficult. As mentioned, adding additional fit parameters will make the fit even more ill conditioned. We will use these expressions to estimate the shift of the magnetic center in the NLC quadrupole magnets in the next section.

\section{THE NEXT LINEAR COLLIDER MAIN LINAC}

The Next Linear Collider main linac utilizes a FODO array in which the space between quadrupoles is occupied with accelerator structures operating at a frequency of $11.424 \mathrm{GHz}$. Each structure is $1.8 \mathrm{~m}$ in length, and three consecutive structures are installed on a common girder. The spacing between quadrupoles is $6 \mathrm{~m}$ at the low energy end of the linac, increasing to 12 and then to $18 \mathrm{~m}$; this permits 1,2 , or 3 girders of rf structures per quadrupole. The betatron phase advance is roughly $90^{\circ}$ per cell although, in the first $35 \%$ of the accelerator, the betatron phase advance per cell is gradually reduced from upstream to downstream.

The linac contains approximately 750 quadrupoles, each of which has a strip line beam position monitor captured in its bore, and approximately $5000 \mathrm{rf}$ structures $[16,17]$. The quadrupoles have an aperture radius of $6.35 \mathrm{~mm}$, corresponding to a $0.5 \mathrm{in}$. full bore, and a maximum pole-tip field of $9 \mathrm{kG}$. In addition, the present magnet design calls for solid cores rather than laminations. This choice has been made to improve the structural integrity of the magnet and increase its inductance, which will lengthen the field decay time and ease the machine protection system requirements. The horizontal betatron functions in the Next Linear Collider main linac are shown in Fig. 2. The linac operates in a pulsed mode, with one train of bunches accelerated per linac pulse and 120 linac pulses per second.

With the exception of dipoles for fast feedback $(120 \mathrm{~Hz})$ and high-bandwidth kickers for shaping the trains of bunches, the NLC main linac contains no steering correctors. The steering of the accelerator is accomplished by remote-controlled translation stages which can position each quadrupole, as well as each end of the rf girders, in both vertical and horizontal. The operational concept for the main linac calls for continually positioning the magnets and $\mathrm{rf}$ girders such that the rms beam offset from the center of the quadrupoles and girders is minimized. In order to accomplish this, it is necessary to measure the offset between the BPM electrical centers and the quadrupole magnetic centers with micron accuracy; however, the offset of a strip-line-type BPM mounted in the bore of a quadrupole is expected to be 100-200 $\mu \mathrm{m} \mathrm{rms} \mathrm{[3].} \mathrm{In} \mathrm{order} \mathrm{to} \mathrm{achieve} \mathrm{the} \mathrm{desired}$ main linac performance, it will therefore be necessary to measure the BPM offsets of the main linac quadrupoles via beam-based techniques to the level of $1-2 \mu \mathrm{m}$. 


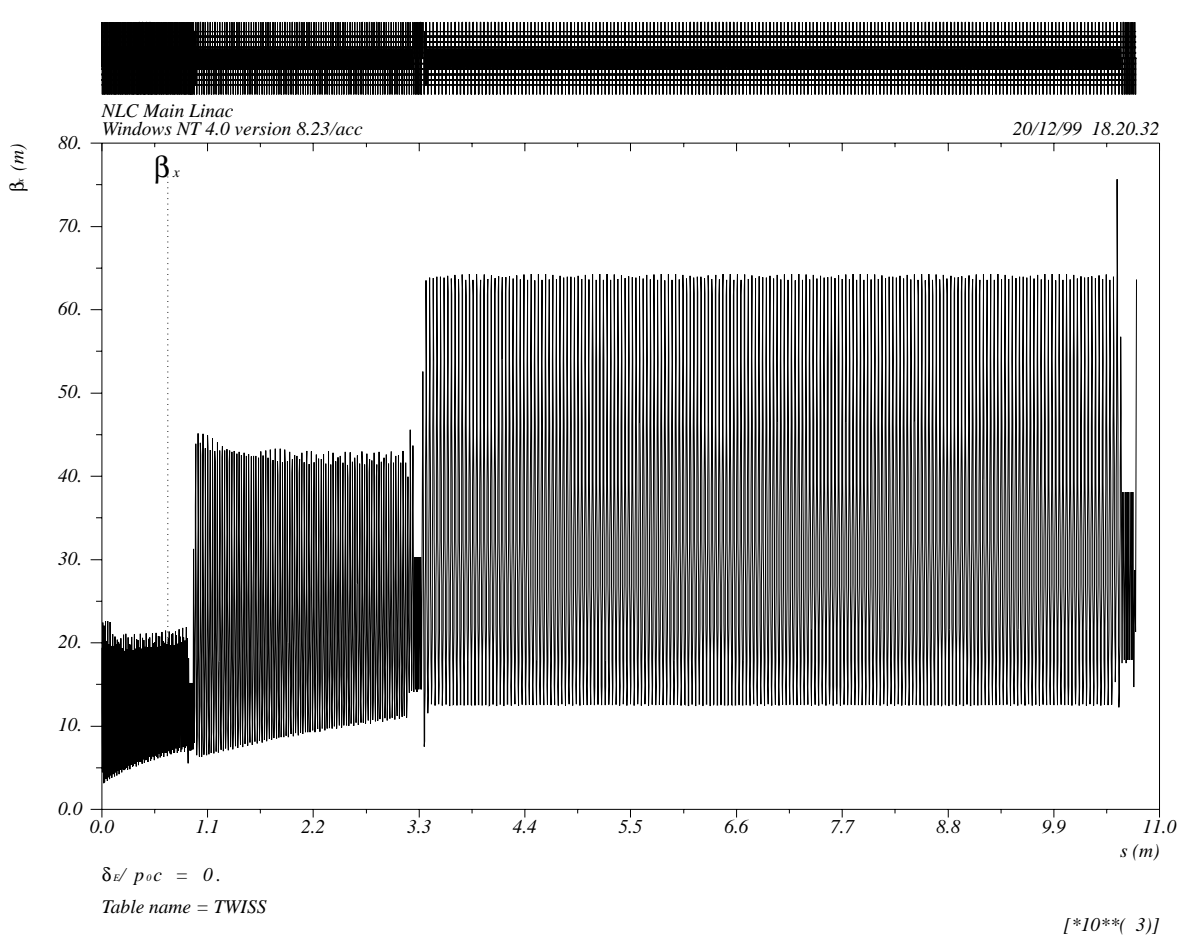

FIG. 2. Horizontal betatron function of the NLC main linac.

In the following sections, we will estimate the statistical resolution for the NLC alignment algorithm and then consider the systematic resolution limit. To estimate the systematic limitations, we will first evaluate the simple analytic expressions, derived in the previous section, to obtain an approximation for the variation of the magnetic center. Then, we will use the measured variation in gradient data from the FFTB [18] to model the NLC magnets and simulate the effect on the beam-based alignment procedures.

\section{A. Statistical resolution in the NLC main linac}

Equation (3) permits us to estimate the statistical resolution achievable in the NLC main linac. The expected BPM resolution is $1 \mu \mathrm{m}$, which has been achieved at the NLC design bunch charge $\left(1 \times 10^{10}\right.$ electrons/positrons $)$ by an existing system [3]; we assume that, during the beam-based alignment operation, each bunch train is reduced to a single bunch with this intensity. For speed of operation, we limit data acquisition to 144 pulses per magnet strength step, which at full machine rate can be acquired in $1.2 \mathrm{~s}$. The number of strength steps is chosen to be five. We will consider cases in which the quad is varied symmetrically about its operating point $(j \in$ $\{-2,-1,0,1,2\})$ and cases in which the quad is varied asymmetrically from its operating point $(j \in\{0,1,2,3,4\}$ or $j \in\{0,-1,-2,-3,-4\})$; the latter option is likely to be required for quads which are operating near their maximum or minimum strength.
As mentioned in the previous section, the phase advance per cell is tapered in the first two regions of the NLC main linac. As a result, the value of $\mathcal{R}_{2}$ varies monotonically from the beginning to the end of these regions. In addition, the value of $\mathcal{R}_{2}$ for "in-plane" measurements (measuring the offset of a horizontally focusing quadrupole in the horizontal plane) is larger than the value for "out-plane" measurements (measuring the offset of a horizontally focusing magnet in the vertical plane). These two constants, here labeled by $\mathcal{R}_{2 \text { in }}$ and $\mathcal{R}_{\text {2out }}$, are tabulated at the beginning and end of each main linac region in Table I. Note that in the analysis that follows we compute only the resolution for horizontally focusing quadrupoles; since the phase advance in the vertical is only slightly different from that in the horizontal, the two are expected to be nearly equivalent.

The remaining free parameter in Eq. (3) is the number of betatron half-wavelengths over which BPM data is

TABLE I. Values of $\mathcal{R}_{2 \text { in }}, \mathcal{R}_{\text {2out }}$, and $K_{q}$ at the beginning and end of each main linac region.

\begin{tabular}{lccc}
\hline \hline Region & $\mathcal{R}_{\text {2in }}\left(\mathrm{m}^{2}\right)$ & $\mathcal{R}_{\text {2out }}\left(\mathrm{m}^{2}\right)$ & $K_{q}\left(\mathrm{~m}^{-1}\right)$ \\
\hline 1, start & 536 & 63 & 0.2424 \\
1, end & 908 & 249 & 0.1683 \\
2, start & 2335 & 232 & 0.1263 \\
2, end & 2742 & 622 & 0.0965 \\
3, start & 5006 & 824 & 0.0737 \\
3, end & 5002 & 831 & 0.0737 \\
\hline \hline
\end{tabular}


TABLE II. Maximum fractional quad strength excursions, $j_{\text {max }} d K_{q} / K_{q}$, required to achieve $1 \mu \mathrm{m}$ statistical resolution of quadrupole misalignments. Fractional strengths are given in percentage of the design strength required. Both symmetric scans about the quadrupole design strength ("Symm") and asymmetric scans in which the quad is scanned monotonically away from its design strength ("Asym") are considered.

\begin{tabular}{lcccc}
\hline \hline & \multicolumn{2}{c}{ In-plane } & \multicolumn{2}{c}{ Out-plane } \\
Region & Symm (\%) & Asym (\%) & Symm (\%) & Asym (\%) \\
\hline 1, start & 0.42 & 0.49 & 1.23 & 1.42 \\
1, end & 0.47 & 0.54 & 0.89 & 1.03 \\
2, start & 0.40 & 0.46 & 1.25 & 1.44 \\
2, end & 0.46 & 0.54 & 0.98 & 1.13 \\
3, start & 0.45 & 0.52 & 1.11 & 1.29 \\
3, end & 0.45 & 0.52 & 1.11 & 1.28 \\
\hline \hline
\end{tabular}

acquired. It is known that, in the NLC main linac, the response functions for beam transport are given by the linear quadrupole optics for short distances, but become dominated by wakefield amplification over long distances. To avoid the wakefield effects, which make the modeling more difficult, we limit the number of half-wavelengths to five.

Table II shows the maximum relative excursion in quadrupole strength, $\Delta K_{q}$, which is required to achieve $1 \mu \mathrm{m}$ statistical resolution at various points in the linac under the conditions outlined above. For in-plane resolution the maximum required quadrupole excursion does not exceed $0.54 \%$, while for out-plane resolution the maximum needed quadrupole excursion is $1.44 \%$. Such small changes in the main linac optics are believed to be well below the level at which the beam quality is degraded. In practice, the alignment algorithm is likely to use maximum quadrupole strength excursions many times those listed in order to ensure that the uncertainty in quadrupole strength is small compared to the change in quadrupole strength; in addition, reducing the statistical error on the measured BPM-to-quad offset will allow the tolerances on other errors to be relaxed.

\section{B. Systematic resolution limit}

As discussed in the previous section, there are many sources of error which could cause a shift in the magnetic center. In this section, we will first estimate the magnetic center variation using the expressions derived in the previous section and then make an estimate based on the measured fields in the FFTB quadrupole magnets. Finally, using this FFTB data, we will evaluate the performance of the algorithm in more detailed simulations.

For the NLC quadrupoles the core efficiency is roughly $90 \%$, and the best expected variation in the permeability in a lot of steel is roughly $0.1 \%$. In this case, Eq. (14) indicates that $\Delta y \approx 0.2 \mu \mathrm{m}$ between the mechanical and magnetic centers of the quadrupole. Since saturation becomes an important effect near the full excitation of the magnet, we expect that the full $0.2 \mu \mathrm{m}$ offset will be realized only by magnets operated near their maximum attainable strength.

The NLC quadrupole magnets are designed to have a temperature rise of $20{ }^{\circ} \mathrm{C}$ in each of the four coils at the nominal operating current, and the magnets are roughly $25 \mathrm{~cm}$ square. The value of $C_{T}$ for steel varies from $1.1 \times 10^{-5}$ to $1.8 \times 10^{-5}$ [19]; using the latter value to attain a worst-case estimate, Eq. (16) shows that this corresponds to $\Delta y \sim 20 \mu \mathrm{m}$ between zero current and full excitation. Similarly, comparison between Eqs. (15) and (16) shows that the effect of differential expansion will be smaller than the effect of overall expansion of the magnet by a factor of $\left[0.425\left(\Delta T_{p}\right)_{\mathrm{rms}} L_{p}\right] /\left[\Delta T_{f} L_{m}\right]$. If we limit the rms coil temperature difference to $20 \%$ of the average temperature rise, and assuming that $L_{p} / L_{m} \approx$ 0.25 , we find that the magnetic center motion due to this effect is $\Delta y \approx 0.5 \mu \mathrm{m}$ between zero current and full excitation. Both of these effects are proportional to $\Delta I / I$, and we have assumed that the magnet achieves thermal equilibrium at each current in the estimates above. For perturbative quadrupole variations $(\Delta I / I<0.1)$ which are expected to require approximately $20 \mathrm{~s}$ per magnet step, we expect the actual change in quadrupole magnetic center due to thermal effects to be small.

Based on the estimates above, we see that the primary effect of concern to the NLC is unequal permeability of the magnet pole pieces. While this effect produces only a $0.2 \mu \mathrm{m}$ offset of the magnetic center, Eq. (7) indicates that such shifts result in a fitting error which is larger than the offset shift by a factor of $K_{q} / d K_{q}$. Therefore, systematic fitting errors of $2-4 \mu \mathrm{m}$ are not inconceivable from this source.

\section{FFTB measurements}

These estimates of the motion indicate a potential limitation for the NLC. At this point, we will use some recent measurements to confirm the values above. Unfortunately, we do not have measurements of the individual pole-tip fields; however, if we assume that the measured quadrupoles are "perfect" and therefore the pole potentials $\Phi_{i}$ are equal, then the $\Phi_{i}$ are linearly proportional to the pole-tip magnetic field, the gradient, and the integrated gradient of the magnet. We can then assemble imperfect quadrupoles by combining pole pieces from different perfect quadrupoles.

For the purposes of this study, we have taken the iron dominated "standard quadrupole" from the FFTB to represent our perfect quadrupoles. The standard quadrupole is assembled from four solid-core iron pole pieces, has an effective length of $46.1 \mathrm{~cm}$, an aperture radius of $1.15 \mathrm{~cm}$, and a peak pole-tip field of $0.97 \mathrm{~T}$. A total of 29 standard quadrupoles were constructed for the FFTB project by the Budker Institute of Nuclear Physics in Russia. Magnetic measurements of the standard quads indicated that all of 
the magnets were matched in gradient at the level of $0.1 \%$ rms variation over the full range of excitation currents $(15$ to $240 \mathrm{~A})$, and that all harmonics were below the $0.1 \%$ level at $70 \%$ of the aperture. The FFTB standard quads represent a recent set of state-of-the-art iron-dominated quadrupoles, which can provide insight into the behavior of such magnets for the NLC.

Our algorithm for estimating the systematic errors in quadrupole variation measurements was the following: each "imperfect" model quad was generated by selecting at random four FFTB quadrupoles to represent the four pole pieces of the imperfect quad; we fit the measured integrated gradient versus current from each FFTB magnet with a polynomial of order 5 and these polynomials were then used to estimate the pole-tip fields for each model magnet; we then computed the values of $x_{j}$ in Eq. (7) as a function of excitation current using Eq. (11), and subtracted the value of $\Delta y$ at the operating point so as to force $x_{j=0} \equiv 0$; finally, we used Eq. (7) to compute $x_{M}-x_{D}$ for the generated magnet.

This algorithm was executed to generate $1000 \mathrm{im}$ perfect magnets and computed the rms of the quantity $x_{M}-x_{D}$. Finally, we took into account the fact that each FFTB quad is constructed of four imperfectly matched pole pieces by doubling the rms of $x_{M}-x_{D}$, and we used this final quantity as our figure of merit for the magnets. In doubling the rms, we have assumed that the matching of pole pieces within an FFTB quad is approximately as good as the matching of the quads to one another. The rms motion of the magnetic center in our 1000 model quadrupoles is plotted as a function of excitation in Fig. 3.

Figures 4 and 5 show the expected rms alignment error as a function of magnet excitation, normalized to the magnet's maximum excitation strength. Figure 4 assumes that the maximum variation in quad strength is $1 \%$ of the operating strength, while in Fig. 5 the maximum variation

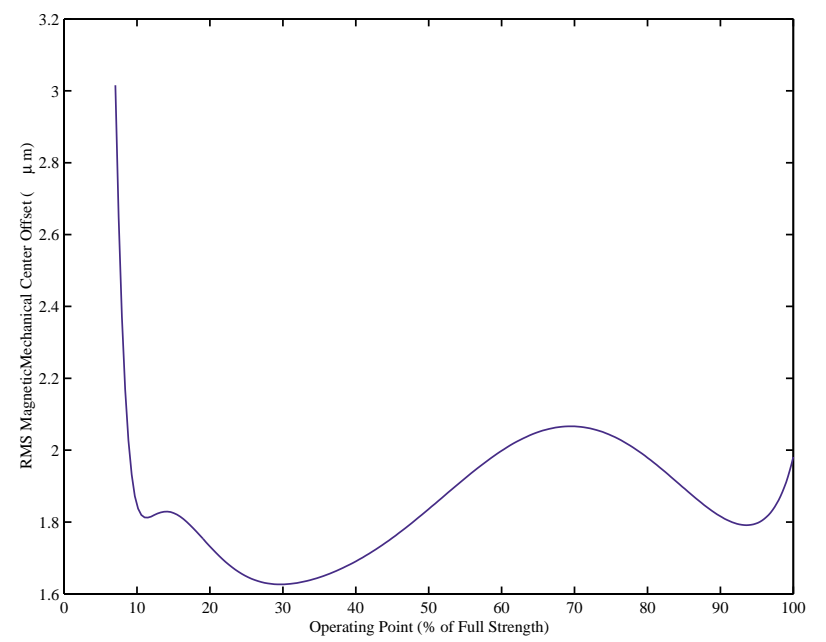

FIG. 3. (Color) rms quadrupole magnetic-mechanical center offset vs excitation using the FFTB magnet model.

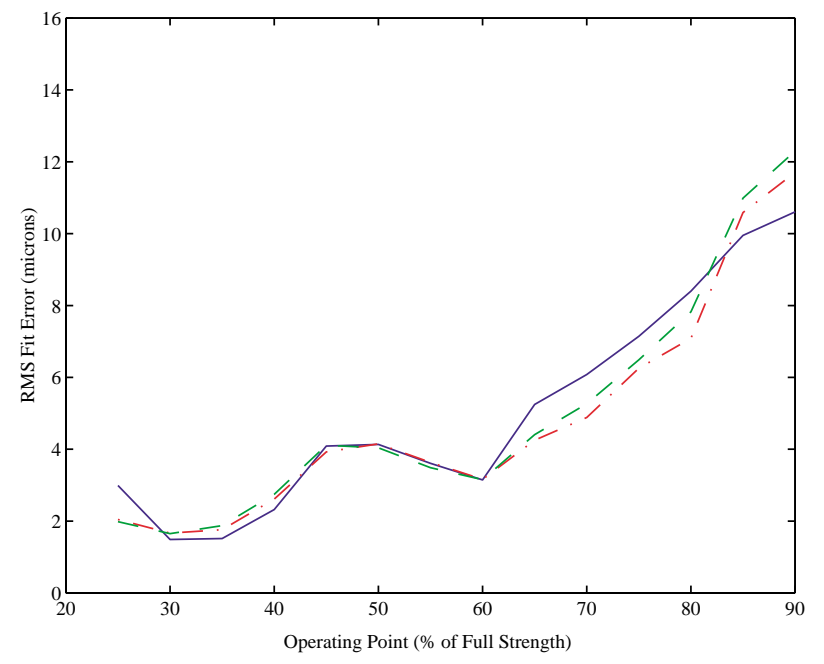

FIG. 4. (Color) rms alignment fit error due to permeability mismatch in NLC main linac quads as a function of design excitation, for maximum strength excursions of $1 \%$ of the design strength. In each case, five values of the quad strength are used. Scans which are symmetric about the operating point (blue solid line), monotonically towards zero strength (red dotdashed line), and monotonically away from zero strength (green dashed line) are shown.

is $10 \%$. In each case, five steps of the quad strength are used, and we compare symmetric scans with asymmetric "up" and "down" scans, in which the quad is stepped monotonically away from its design strength. While the NLC design calls for measuring the quadrupole to BPM center offset with an rms error of 1 to $2 \mu \mathrm{m}$, Figs. 4 and 5 indicate that the achieved error will be 2 to $14 \mu \mathrm{m}$, substantially larger. Note that the rms systematic error

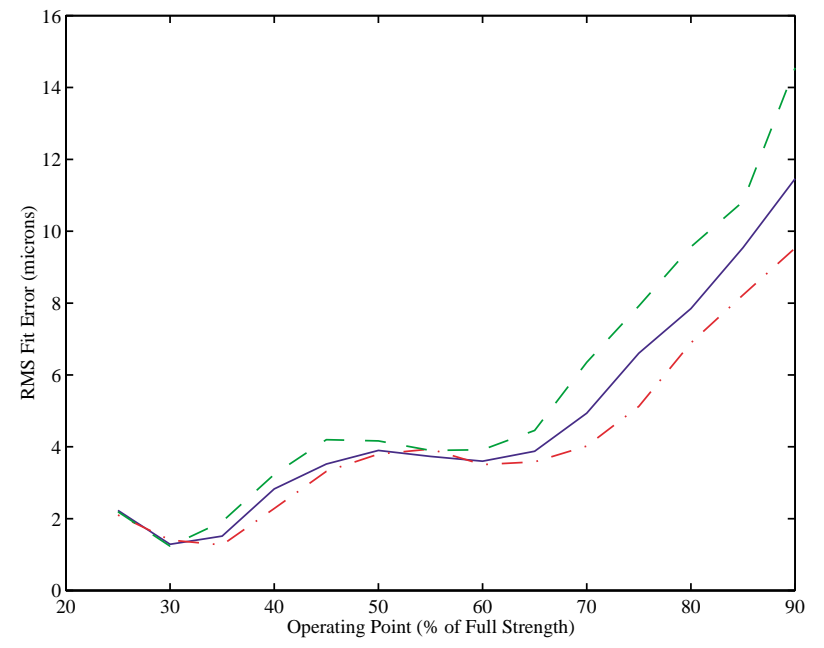

FIG. 5. (Color) rms alignment fit error due to permeability mismatch in NLC main linac quads as a function of design excitation, for maximum strength excursions of $10 \%$ of the design strength. The three curves are defined identically with Fig. 4. Note the similarity in amplitude and overall shape for $1 \%$ and $10 \%$ maximum excursion. 
as a function of design excitation is nearly the same for $1 \%$ maximum variation as for $10 \%$. For such small total variations, the second term on the right-hand side (rhs) of Eq. (7) is dominant, and $x_{j}$ is dominated by the linear motion of the quadrupole center as a function of excitation. Therefore, as $K_{q} / d K_{q}$ is reduced for increased values of $d K_{q}$, the $x_{j}$ values are increased, and to lowest order the second rhs term in Eq. (7) remains constant.

Figure 6 shows the precision that is achievable if a five step quad scan is performed in which the maximum variation is allowed to increase. We consider quads which have a nominal operating point that is $70 \%, 80 \%$, or $90 \%$ of the maximum strength of the FFTB magnets. Figure 6 shows that we can achieve micron accuracy of the fit if we permit the magnets to be reduced in strength by up to $90 \%$ of their design strength. In this case the quadrupole variation procedure approximates reducing the quad strength to zero, which improves the robustness of the procedure. It remains to be determined whether other factors such as the resulting downstream beam size will prohibit such large variations in quad strength or whether other systematic errors will arise in such nonperturbative measurements which will eliminate the gains described above.

In the case in which both $x_{D}$ and $\dot{x}$ are simultaneously fitted, Eq. (9) relates the fit resolution to the parameters of the scan and the data acquisition system. Equation (9) can be rewritten as follows:

$$
\begin{gathered}
\sigma_{x}=\sigma_{\mathrm{BPM}} F_{1} F_{2}, \quad \text { where } F_{1} \equiv\left(m n \mathcal{R}_{2} K_{q}^{2}\right)^{-0.5} \\
F_{2} \equiv \sqrt{\frac{\mathcal{K}^{2} \sum_{j} j^{4}+2 \mathcal{K} \sum_{j} j^{3}+\sum_{j} j^{2}}{\mathcal{K}^{4}\left[\sum_{j} j^{4} \sum_{j} j^{2}-\left(\sum_{j} j^{3}\right)^{2}\right]}}
\end{gathered}
$$

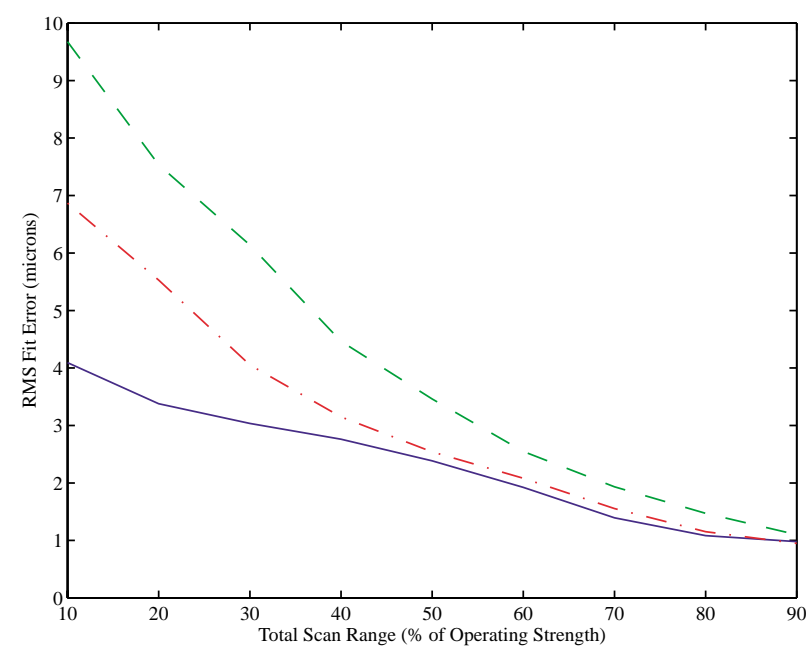

FIG. 6. (Color) rms alignment fit error due to permeability mismatch as a function of maximum strength excursion. In this case, five values of the quad strength are used, and the quad is stepped monotonically towards zero strength. Curves shown are for a design strength which is $70 \%$ (blue solid line), $80 \%$ (red dot-dashed line), or $90 \%$ (green dashed line) of the maximum strength of the quad.

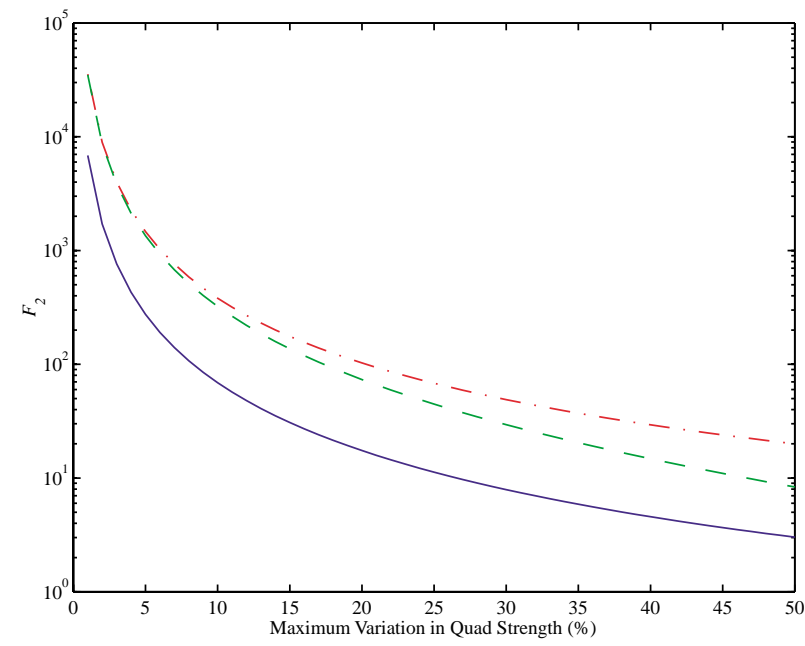

FIG. 7. (Color) $F_{2}$ as a function of $\Delta \mathcal{K}$. Symmetric scans (blue solid line), monotonic scans towards zero strength (green dashed line), and monotonic scans away from zero strength (red dot-dashed line) are shown.

Figure 7 shows the value of $F_{2}$ as a function of the maximum quadrupole strength excursion $\Delta \mathcal{K} \equiv j_{m} \mathcal{K}$ for symmetric and monotonic quad scans utilizing five steps per scan. Values in Table I were used to evaluate $F_{1}$ for the NLC main linac, yielding in-plane values of $F_{1}$ from $6.1 \times 10^{-3}$ to $7.4 \times 10^{-3}$, while out-plane values vary from $1.4 \times 10^{-2}$ to $1.9 \times 10^{-2}$, assuming values of $m$ and $n$ identical to those in Sec. VA. In order to achieve a resolution of $1 \mu \mathrm{m}$ in the in-plane measurements, a maximum quad variation of $7 \%$ for symmetric scans or $17 \%$ for scans monotonically away from zero strength is required; for out-plane measurements, the required variation is $11 \%$ or $28 \%$ for the same types of scans. These values are more than an order of magnitude larger than the strength excursions required to fit $x_{D}$ in the

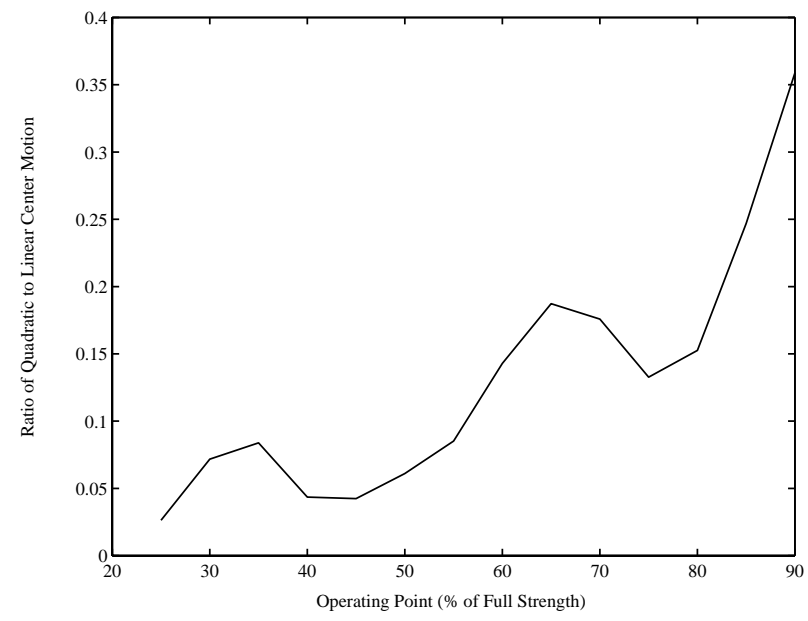

FIG. 8. rms ratio of $\ddot{x}$ to $\dot{x}$ for 1000 randomly generated ironcore quadrupoles. Data were generated for five step symmetric scans with $\Delta \mathcal{K}$ of $10 \%$. 


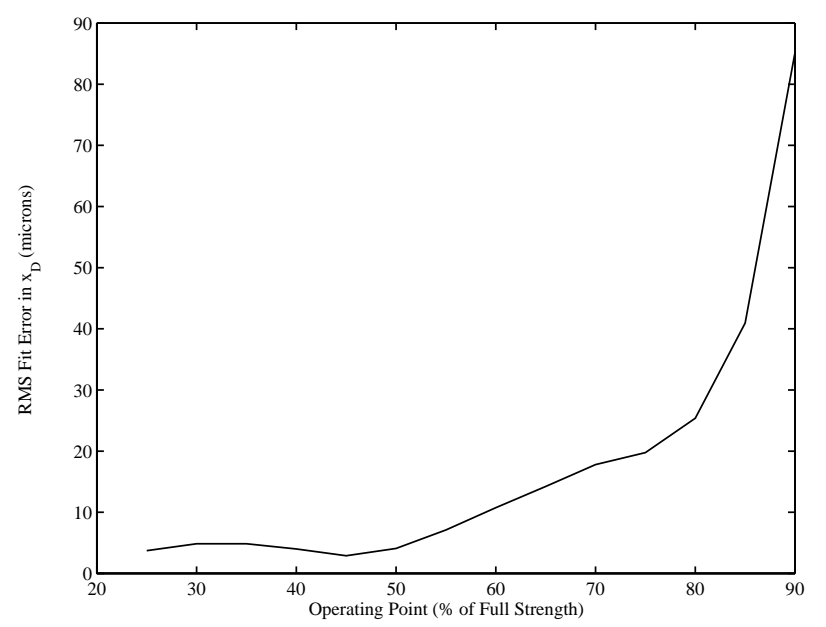

FIG. 9. rms fit error on $x_{D}$ when $x_{D}$ and $\dot{x}$ are fitted, but $\ddot{x}$ is ignored, for the solid-core quad modeled in the text. The case shown is a symmetric five step scan in which the maximum excursion is $10 \%$ of the operating strength.

one-parameter model described in Eq. (3). We conclude that the error ellipse for the two-parameter fit is extremely elongated.

The resolutions quoted above are valid only if the quadratic motion of the quad center $\ddot{x}$ is small compared to the linear motion. As mentioned before, if $\ddot{x} / \dot{x}>\mathcal{K}$, the quadratic deflection measured on downstream BPMs will be dominated by $\ddot{x}$, which will ultimately result in an incorrect fitted value of $x_{D}$. Figure 8 shows the rms value of $\ddot{x} / \dot{x}$ for 1000 magnets generated as described above, assuming a symmetric scan of five steps from $10 \%$ below the design strength to $10 \%$ above the design strength. Note that $\ddot{x} / \dot{x}$ is typically larger than 0.05 , which is the value of $d K_{q} / K_{q}$, and in fact rises to almost 0.4 . This indicates that the two-parameter fit cannot be used. Figure 9 shows the rms systematic error in fitting $x_{D}$ which occurs in such a two-parameter fit using the magnet modeling described above and a symmetric scan with maximum excursion of $10 \%$. A comparison of Figs. 9 and 5 indicates that the systematic error will be smaller if the center motion is ignored than if an attempt is made to include it in the fit.

\section{CONCLUSIONS}

Beam-based alignment of quadrupoles is becoming a key commissioning procedure for high performance accelerators. We have estimated the limitations which arise in the subset of beam-based alignment techniques in which quadrupoles are varied in strength but not deactivated entirely, and downstream deflections are measured to determine the offsets of the quads. These algorithms for beam-based alignment are limited by a combination of statistical and systematic errors. The statistical limit can be estimated analytically from the parameters of the quadrupole scan, the lattice, the BPM system, and the amount of data which is to be acquired. For a given amount of BPM data, the optimum resolution is obtained by a symmetric scan of the quad about its operating point in which the number of steps of the quad strength is minimized and the size of the strength steps is maximized.

The most serious systematic error is variation of the quadrupole magnetic center which occurs when the strength is changed. The systematic error can be estimated analytically if the motion of the center on each step can be determined. The effect becomes quite important because the sensitivity to the motion of the quadrupole center is amplified by the inverse of the fractional quadrupole variation $d K_{q} / K_{q}$ which one would like to keep small to limit the perturbation to the focusing lattice. The error can be minimized by varying the quadrupole strength monotonically towards zero and by approaching zero strength as closely as the beam line will permit. If the motion of the magnetic center is linearly proportional to the strength, it is, in principle, possible to fit for the linear motion and the beam-to-quad offset at the design strength, though the errors tend to be large because the fit is ill conditioned. Furthermore, this multiparameter fit may not be possible if the motion of the magnetic center is a nonlinear function of strength.

In applying the statistical and systematic limits to the case of the NLC main linac, we found that it was easy to achieve submicron statistical resolution with reasonable choices for the parameters, but the systematic errors due to the magnetic center shift could be several times larger. In order to reduce the systematic errors to the level of the statistical, it would be necessary to vary the magnet strength monotonically towards zero and increase the strength step size significantly. Such a procedure cannot be considered a perturbative measurement and may be limited by the resulting large changes in linac beam dynamics. In addition, for the FFTB quadrupole model, it appears that the nonlinear motion of the magnetic center with the excitation was too large to permit a simultaneous fit of the linear motion and the beam-to-quad offset at the design strength.

In the event that both the nonperturbative quad scan and the two parameter fit prove impractical, there are several additional engineering solutions which might permit quadrupoles in future accelerators to be aligned with the high precision and accuracy discussed above. These include constructing quads from laminations rather than as solid-core magnets or improving the permeability matching between magnet pole pieces, designing the magnets to operate well below saturation levels, and limiting the temperature variation in the magnets. Alternately, one could consider developing a program to fiducialize the BPMs to the quadrupole magnets with high accuracy prior to installation or developing in situ fiducialization techniques which do not rely on beam measurements. These latter techniques would require substantial development to ensure that the fiducialization tolerances can be met. In 
addition, an expanded program of measurements of the magnetic center motion as a function of excitation strength will be needed to verify the expected errors and to determine the viability of any of these approaches.

\section{ACKNOWLEDGMENTS}

The ideas, suggestions, and assistance of C. Adolphsen, G. Bowden, T. Slaton, and M. D. Woodley were invaluable in performing the studies described above. This work was performed under Department of Energy Contract No. DE-AC03-76SF00515.

[1] P. Emma, R. Carr, and H. D. Nuhn, Nucl. Instrum. Methods Phys. Res., Sect. A 429, 407 (1999).

[2] K. Kubo, SLAC Technical Report No. LCC-Note-0016 (unpublished).

[3] S. Smith, P. Tenenbaum, and S.H. Williams, Nucl. Instrum. Methods Phys. Res., Sect. A 431, 9 (1999).

[4] P. Emma, in Proceedings of the European Particle Accelerator Conference, Berlin, 1992, edited by H. Henke, H. Homeyer, and C. Petit-Jean-Genaz (Editions Frontíeres, Gif-sur-Yvette, 1992), pp. 1625-1627.

[5] T. L. Lavine et al., in Proceedings of the Linear Accelerator Conference, Williamsburg, VA, 1988 (National Technical Information Service, Springfield, 1988), pp. 646-648.

[6] T. Raubenheimer and R. Ruth, Nucl. Instrum. Methods Phys. Res., Sect. A 302, 191 (1991).

[7] R. W. Assmann et al., in Proceedings of the International Workshop on Accelerator Alignment, Tsukuba, Japan, 1995 (KEK, Tsukuba, Japan, 1995), pp. 463-477.
[8] F. J. Decker et al., in Proceedings of the European Particle Accelerator Conference, Stockholm, 1998, edited by S. Myers et al. (Institute of Physics, Bristol, 1998), pp. $454-456$.

[9] P. Tenenbaum et al., in Proceedings of the Fourth International Workshop on Accelerator Alignment (IWAA 95), Tsukuba, Japan, 1995 (KEK, Tsukuba, Japan, 1995), pp. 393-402.

[10] R. Assmann et al., in Proceedings of the IEEE Particle Accelerator Conference, Vancouver, Canada, 1997, edited by M. Comyn, M. K. Craddock, M. Reiser, and J. Thomson (IEEE, Piscataway, NJ, 1997), pp. 470-472.

[11] T. O. Raubenheimer and D. Schulte, in Proceedings of the IEEE Particle Accelerator Conference, New York, 1999, edited by A. Luccio and W. MacKay (IEEE, Piscataway, NJ, 1999), pp. 3441-3443.

[12] I. Barnett et al., in Proceedings of the International Workshop on Accelerator Alignment, Tsukuba, Japan, 1995 (Ref. [7]), pp. 421-426.

[13] C. Adolphsen and T. O. Raubenheimer, in Proceedings of the IEEE Particle Accelerator Conference, Washington, DC, 1993 (IEEE, Piscataway, NJ, 1993), pp. 417-419.

[14] J. Irwin et al., Phys. Rev. Lett. 82, 1684 (1999).

[15] K. Halbach, Nucl. Instrum. Methods 74, 147 (1969).

[16] C. Adolphsen et al., A Zeroth-Order Design Report for the Next Linear Collider (SLAC, Stanford, CA, 1996), p. 343.

[17] Y. Nosochkov and T. O. Raubenheimer, SLAC Technical Report No. LCC-Note-0024 (unpublished).

[18] V. Balakin et al., Phys. Rev. Lett. 74, 2479 (1995).

[19] E. Avalonne and T. Baumeister, Marks' Handbook for Mechanical Engineers (McGraw-Hill, New York, 1987), pp. 6-11. 